\title{
RESPUESTAS FISIOLOGICAS, RESISTENCIA AL ESTRES Y CRECIMIENTO DE PLANTAS DE Eucalyptus globulus Y Eucalyptus grandis MODIFICADOS POR POTASIO BAJO CONDICIONES DE SEQUÍA
}

\author{
Guarnaschelli, Ana B. ${ }^{6}$, Chiavassa, Juan A. ${ }^{1}$ y Garau, Ana M. ${ }^{1}$
}

\section{RESUMEN}

Las sequías y las heladas son los factores de estrés que más comprometen la fisiología y el crecimiento de las plantas forestales, el estrés hídrico y las bajas temperaturas luego de la plantación, ocasionan serios daños y afectan el comportamiento de las plantas durante el establecimiento. Desafortunadamente, en los escenarios de cambio climático se predice que la influencia de tales eventos se incrementará, lo cual supone un gran desafío para la producción forestal.

Algunas prácticas de vivero pueden minimizar los efectos negativos de las condiciones de sequía y bajas temperaturas, y mejorar el comportamiento de las plantas luego de la plantación. Por ejemplo, la fertilización potásica durante el período de rustificacion en vivero resulta importante para la nutrición de la planta y también cumple un rol relevante aliviando el estrés. Sin embargo, es necesario generar aún más información sobre la influencia de estas prácticas en especies de Eucalyptus y sobre cómo podrían ayudar en la superación de estas restricciones ambientales.

Con el objetivo de evaluar algunas respuestas fisiológicas, resistencia al estrés y crecimiento de plantas de Eucalyptus globulus y Eucalyptus grandis, se las sometió a diferentes regímenes de riego y niveles de fertilización potásica. Las plantas control (C) se regaron diariamente, mientras que las moderadamente estresadas (S1) y las severamente estresadas (S2) se regaron cada seis y nueve días, respectivamente. En cada nivel de riego, la mitad de las plantas recibió fertilización potásica $(\mathrm{K} 1)$ y las restantes no fueron fertilizadas (K0). Para evitar el acceso eventual de agua de precipitación, las plantas se cubrieron con un techo de polietileno rebatible.

El experimento se estableció bajo condiciones controladas y durante cinco meses (desde julio a diciembre de 2009) se midieron periódicamente el diámetro a la altura del cuello y la altura, y se calcularon las respectivas tasas de crecimiento relativo. Se evaluaron diversas características fisiológicas y tolerancia a la sequía a través de mediciones de conductancia estomática, contenido relativo de agua, potencial agua y variables hídricas. La tolerancia al frío se evaluó a través de la presencia de antocianas en los tejidos foliares, la cual se correlaciona con la severidad de la fotoinhibición inducida por frío. Las respuestas de las especies se evaluaron además a través de un índice de plasticidad fenotípica.

La sequía modificó la mayoría de las características de las plantas y redujo el crecimiento. Las plantas que crecían bajo los niveles S1 y S2 mostraron menor conductancia estomática, potencial agua y contenido relativo de agua. El potasio generó un efecto significativo, incrementando el contenido relativo de agua de las plantas S1K1 y S2K1. En respuesta al estrés hídrico las plantas desarrollaron ajuste osmótico y ajuste elástico. La magnitud promedio de ajuste osmótico en las plantas $\mathrm{S} 1$ y $\mathrm{S} 2$ fue $0,10 \mathrm{MPa}$ y $0,20 \mathrm{MPa}$ respectivamente, pero solo las plantas S2 mostraron un incremento en la elasticidad de 2,2 MPa. E. grandis tuvo menor potencial osmótico y menor elasticidad. Los niveles de antocianas fueron mayores en las plantas $\mathrm{C}$ que en las planta S1 y S2, particularmente en E. grandis, mientras que en E. globulus se observaron pequeñas diferencias entre los tres regímenes hídricos. El diámetro y la altura fueron menores en las plantas con estrés hídrico, especialmente, en las que crecían bajo condiciones S2. E. grandis

6 Departamento de Producción Vegetal, Facultad de Agronomía, Universidad de Buenos Aires. Argentina. guarnasc@agro.uba.ar 
tuvo mayores tasas de crecimiento que E. globulus. Los índices de plasticidad mostraron que el crecimiento en diámetro fue menos afectado por la sequía que el crecimiento en altura y también mostraron mayores reducciones en el crecimiento de E. grandis.

Estos resultados indican que las dos especies manifiestan mecanismos de tolerancia a la sequía que les permiten soportar el estrés, pero confirman también que la sequía y el frío afectan el crecimiento de las plantas de E. globulus y E. grandis. Las plantas sometidas a sequía mostraron menores niveles de fotoinhibición, lo que sugiere que los ajustes en la tolerancia a la sequía las habrían protegido de las bajas temperaturas. El agregado de $\mathrm{K}$ tuvo efecto positivo protegiendo a las plantas que crecían bajo sequía. Las plantas estresadas del nivel K1 mostraron mayor contenido de agua, lo que implica que la fertilización potásica es una herramienta útil para los productores de plantas de Eucalyptus, aportando calidad de las mismas y con potencial efecto positivo luego de la plantación. Pero no fue posible establecer su efecto sobre la protección frente a las bajas temperaturas.

Palabras clave: Eucalyptus globulus, Eucalyptus grandis, Resistencia a Sequía y a Heladas, Fertilización en Viveros

\section{SUMMARY}

Droughts and frosts are among the most frequent stress factors that compromise physiology and growth of tree seedlings. Water stress and low temperatures soon after planting cause serious damage altering plant performance during the establishment. Unfortunately the influence of such events is predicted to increase under the present climate change scenario, which supposes a great change for forest production.

Some nursery practices may contribute to minimize the detrimental effects of drought and cold conditions and improve plant behavior after plantation. For example, potassium fertilization during the nursery hardening period is not only important for plant nutrition, but also it has a relevant role alleviating stress. However, it is still necessary to generate more information about the influence of these practices on Eucalyptus species and how they could allow overcoming theses environmental constraints.

With the aim to evaluate some physiological responses, stress resistance and growth of Eucalyptus globulus and Eucalyptus grandis seedlings plants were submitted to different water regimes and potassium levels. Control plants (C) were irrigated daily while moderate stressed (S1) and severe stressed plants (S2) were irrigated every other six-days and nine-days, respectively. At each water regime, half of the plants received potassium fertilization (K1) and the rest were not fertilized $(\mathrm{K} 0)$. To avoid the access of water from rainfall plants were covered when necessary with a removable polyethylene roof.

The experiment was established under controlled conditions and during five months (from July to December 2009) root collar diameter and height were measured periodically and their respective relative growth rates were calculated. Several physiological characteristics and drought tolerance were assessed through measurements of stomatal conductance, water potential, relative water content and tissue water relations, while frost tolerance was evaluated through the presence of anthocyanins in foliage, which are correlated to the severity of cold-induced photoinhibition. Species responses were analyzed applying an phenotypic plasticity index.

Drought modified most of the plant characteristics and reduced seedlings growth. Plants growing under S1 and S2 conditions showed lower stomatal conductance, water potential and relative water content. Potassium had also significant effect, increasing relative water content of S1K1 and S2K1 plants. As responses to water stress, plants developed osmotic and elastic adjustment capacity. The average magnitude of osmotic adjustment in S1 and S2 plants was 0.10 $\mathrm{MPa}$ and $0.20 \mathrm{MPa}$, respectively, but only S2 plants showed a significant increase in tissue 
elasticity of 2.2 MPa. E. grandis had lower osmotic potential and tissue elasticity. Anthocyanin levels were higher in C plants than in S1 and S2 plants, particularly in E. grandis, while in E. globulus small differences were observed among the three water regimes. Diameter and height were lower in water stressed plants, specially, in those growing under S2 conditions. E. grandis had higher relative growth rates than $E$. globulus. Indexes of plasticity indicated that diameter growth was less affected by drought than height growth and also showed higher reductions in E. globulus growth.

These results showed that the two species displayed drought tolerance mechanisms that allowed them to withstand stress but confirmed again that both drought and cold affect the growth of $E$. globulus and $E$. grandis seedlings. Plants submitted to the restrictive water regimes showed lower signs of photoinhibition, which suggest that drought tolerance adjustments might have contributed to protect them against low temperate. Potassium addition had positive effect protecting plants that were growing under drought. Stressed seedlings of the K1 level showed higher relative water content, which means that potassium fertilization offers a management tool for Eucalyptus growers concerned with plant stock quality, with potential positive effects after plantation. Although it did not have any evident and direct effect protecting plants from low temperatures.

Key Words: Eucalyptus globulus, Eucalyptus grandis, Droughts and Frosts Resistance, Fertilization in Nursery Production.

\section{INTRODUCCIÓN}

Durante el establecimiento diversos factores de estrés comprometen el crecimiento y la supervivencia de las plantas forestales. Las sequías y las heladas son fenómenos muy frecuentes en el centro y noreste de la Argentina, regiones donde se encuentran distribuidas las plantaciones de eucalipto. A los efectos de estos eventos habituales se suma la amenaza del cambio climático, respecto del cual se predice un aumento en la frecuencia e intensidad de los mismos (IPCC 2007). Una evidencia son los daños en las plantaciones generados por la heladas ocurridas en los últimos años en la provincia de Entre Ríos (Garrán et al., 2007, Garrán y Garín, 2012, INTA, 2012).

Entre las principales especies cultivadas en el país, Eucalyptus globulus ssp. globulus y Eucalyptus grandis destacan particularmente por la calidad de la madera y elevada productividad. Estas dos especies manifiestan moderada resistencia a la sequía pero baja tolerancia a las heladas, particularmente E. grandis (Burguess, 1983; Golfari, 1985; Stoneman, 1994).

Existen herramientas que permiten sortear estas adversidades climáticas, como el uso de material genético tolerante, la adecuación de prácticas durante el establecimiento y técnicas culturales de vivero. Se ha observado variabilidad en la capacidad para tolerar condiciones de estrés por bajas temperaturas entre especies del género Eucalyptus y también entre procedencias de una misma especie (Raymond et al., 1992, Volker et al., 1995, Moraga et al., 2006). En tal sentido, el programa de mejoramiento genético de INTA de E. grandis incluye en la actualidad el desarrollo de clones selectos, incorporando la resistencia al frío como otro de los criterios de selección (Marcó, 1986, Marcó, 2005, Harrand y Marcó, 2012), como así también la generación y selección de clones híbridos interespecíficos (Harrand, 2005), con otras especies del género más tolerantes al estrés, a fin de obtener materiales con una mejor respuesta en área marginales por factores climáticos y/o edáficos. En E. globulus ssp. globulus se están realizando cruzamientos con Eucalyptus dunnii, especie que manifiesta buen comportamiento frente al frío.

Con relación a las deficiencias hídricas, diversos estudios indican que existe variabilidad en tolerancia a la sequía entre especies de Eucalyptus (Merchant et al., 2007, Guarnaschelli et al., 2009), entre procedencias (Tuomela, 1997, Guarnaschelli et al., 2003, Guarnaschelli et al., 2006) y clones de un misma especie (Pita y Pardos, 2001; Costa e Silva et al., 2004).

Adecuar la fecha de plantación, controlar las malezas y fertilizar los cultivos para promover el crecimiento de las plantas, logrando mayores dimensiones y menores riesgos de 
daños por sequía y bajas temperaturas, son estrategias factibles para evitar las condiciones de estrés (Smith et al. 1997; White et al., 2011, Larocca, 2012). Por su parte, Algunas prácticas de vivero pueden minimizar los efectos negativos de las sequías y las bajas temperaturas, mejorando el comportamiento de las plantas luego de la plantación. Por ejemplo, la fertilización potásica durante el período final de aclimatación en vivero resulta no solo importante para la nutrición de la planta sino que también cumple un rol relevante aliviando el estrés. La restricción de riegos, utilizada para aclimatar las plantas a la sequía (Landis et al., 1989; Guarnaschelli et al., 2006), es una práctica también recomendada para aumentar la tolerancia a las bajas temperaturas (Bigras y Dumais, 2005).

El $\mathrm{K}$ es un macronutriente esencial para el crecimiento de las plantas. Desempeña un papel destacado en diversos procesos fisiológicos, tales como la regulación estomática, el traslado de fotoasimilados por el floema, la activación de enzimas y la osmoregulación. Una mayor concentración de electrolitos como el $\mathrm{K}^{+}$dentro del citoplasma contribuye a la osmorregulación. El ajuste osmótico, al aumentar la turgencia de los tejidos, facilita el mantenimiento de la apertura estomática y el funcionamiento del aparato fotosintético ante condiciones de estrés hídrico (Morgan, 1984). Asimismo, el descenso en el potencial osmótico disminuye el punto de congelamiento, reduciendo consecuentemente el riesgo de congelamiento intracelular, la formación de cristales de hielo, la rotura de membranas y la muerte celular (Moraga et al., 2006). En diversos trabajos se ha observado que las plantas que recibieron dosis de $\mathrm{K}$ respondieron mejor al estrés hídrico (van den Driessche, 1992, Garau et al, 2004a, 2004b, Texeira et al., 2008; Guarnaschelli et al., 2010).

Otro aspecto adicional y de gran importancia es que la fertilización potásica puede, además, contribuir a un mejor comportamiento de las plantas frente al estrés biótico (Cakman, 2005; Landis, 2005). El K está involucrado también en mecanismos de defensa contra plagas y enfermedades. La incidencia y severidad de daños por plagas y enfermedades se reduce en plantas bien nutridas, ya que se estimula la síntesis de compuestos fenólicos, generalmente asociados con la defensa de las plantas contra herbívoros y patógenos. Recientemente, Römheld y Kyrkby (2010) destacaron las funciones del K en las plantas y resaltaron la necesidad de explorar el rol de este catión cuando las plantas se encuentran en situaciones de estrés.

Un mecanismos protector frente a los efectos nocivos por fotoinhibición condicionada por déficits hídricos y la exposición a las bajas temperaturas, es la síntesis de antocianas (Nicotra et al., 2003; Gould, 2004; Hughes et al., 2005). Esta capacidad es variable entre especies y podría modificarse por efecto de prácticas culturales (Close et al., 2002).

Por todo lo expuesto, el objetivo del presente trabajo fue estudiar bajo condiciones controladas los efectos de la fertilización potásica en plantas jóvenes de E. grandis y E. globulus ssp. globulus, creciendo en tres regímenes hídricos diferentes, sobre la resistencia a la sequía y a las bajas temperaturas.

\section{MATERIAL Y MÉTODO}

El experimento se desarrolló en el predio de la Cátedra de Dasonomía de la Facultad de Agronomía de la Universidad de Buenos Aires (343'ㄱ' ' S, 58²9'47" O, y 20 msnm) y consistió en un diseño factorial de dos especies por dos niveles de fertilización potásica y tres niveles de disponibilidad hídrica, que se extendió por un período de aproximadamente cinco meses.

La producción de plantas se realizó en la Cátedra de Dasonomía en invernáculo tipo túnel con cobertura de polietileno (150 micrones de espesor) removible. Se utilizó semilla importada de Australia de E. globulus ssp. globulus de origen Jeeralang y de E. grandis de origen Kendall (Cuadro $\mathrm{N}^{\circ} 1$ ). 
Cuadro $\mathrm{N}^{\circ} 1$

UBICACIÓN Y DATOS CLIMÁTICOS DE LOS ORÍGENES DE SEMILLAS

\begin{tabular}{|c|c|c|c|c|c|c|c|}
\hline \multirow{2}{*}{ Especie } & \multirow{2}{*}{ Origen } & \multirow{2}{*}{$\begin{array}{l}\text { Lat. } \\
\text { (S) }\end{array}$} & \multirow{2}{*}{$\begin{array}{l}\text { Long. } \\
\text { (E) }\end{array}$} & \multirow{2}{*}{$\begin{array}{c}\text { Altitud } \\
\text { (msnm) }\end{array}$} & \multirow{2}{*}{$\begin{array}{c}\text { Precipitación } \\
\text { Media } \\
\text { (mm/año) }\end{array}$} & \multicolumn{2}{|c|}{$\begin{array}{c}\text { Temperatura } \\
\left({ }^{\circ} \mathrm{C}\right)\end{array}$} \\
\hline & & & & & & $\begin{array}{l}\text { Max } \\
\text { Media }\end{array}$ & $\begin{array}{c}\text { Min } \\
\text { Media }\end{array}$ \\
\hline E. globulus & Jeerelang VIC & $38^{\circ} 19^{\prime}$ & $146^{\circ} 33^{\prime}$ & 222 & 1.390 & 22 & 8 \\
\hline E. grandis & Kendall NSW & $31^{\circ} 37^{\prime}$ & $152^{\circ} 43^{\prime}$ & 72 & 1.260 & 21 & 13 \\
\hline
\end{tabular}

NSW: New South Wales, Australia

VIC: Victoria, Australia

Las semillas se sembraron en febrero de 2009 en bandejas plásticas con 40 contenedores de $93 \mathrm{~cm}^{3}$ cada uno, utilizando como sustrato una mezcla de corteza de pino comportada y corteza de coco.

A los tres meses fueron trasplantadas a envases de polietileno negro de $3 \mathrm{~L}$, con una mezcla de tierra orgánica y arena en una proporción aproximada de 3:1 (v/v) como sustrato. A principios de junio de 2009 se seleccionaron al azar 72 plantas de cada especie, se dispusieron a la intemperie en una condición de plena disponibilidad de luz y se regaron diariamente.

A principios de julio las plantas de cada especie se separaron en tres grupos semejantes y fueron sometidas a tres regímenes hídricos. Las plantas control (C) se regaron diariamente, mientras que las moderadamente estresadas (S1) y las severamente estresadas (S2) se regaron cada seis y nueve días, respectivamente.

Posteriormente, la mitad de las plantas de cada especie y régimen hídrico fue fertilizada con potasio (K1) y la otra mitad no recibió fertilización (K0). En las plantas K1 se agregó cloruro de potasio $(\mathrm{KCl})$ en una dosis de $80 \mathrm{mg}$ de $\mathrm{K}$ por $\mathrm{dm}^{3}$ de de sustrato. El experimento se extendió hasta inicios de diciembre de 2009.

El comportamiento de las plantas a lo largo del período experimental se evaluó a través de la medición de diversas características fisiológicas, morfológicas y de crecimiento.

El estado hídrico de las plantas se evaluó a través del contenido relativo de agua (CRA) en discos foliares de $0,5 \mathrm{~cm}$ de diámetro siguiendo la metodología de Beaddle et al. (1993).

Para calcular el CRA se utilizó la fórmula:

$$
\begin{array}{cc}
\text { CRA }=(\text { PF-PSe }) /(\text { PSa-PSe }) \times 100 \\
\text { Donde: } \quad \text { PF (peso fresco }) \\
\text { PSa (peso a saturación) } \\
\text { PSe (peso seco). }
\end{array}
$$

Al finalizar el período experimental se midió además el potencial agua foliar $\left(\Psi_{\mathrm{a}}\right)$ a la madrugada y al mediodía con una bomba de presión modelo PMS 600 (PMS Instruments, Corvalis, OR).

La conductancia estomática $\left(g_{s}\right)$ se midió periódicamente al mediodía en hojas jóvenes 
totalmente expandidas, con un porómetro de estado estacionario LI-1600 (Li-Cor Inc., Lincoln, NE).

Las variables hídricas se midieron a través de las curvas de presión-volumen -CPV(Ritcher, 1978) en porciones apicales de tallo con 3-4 pares de hojas, previa hidratación. El $\Psi_{\text {a }}$ se medió con la bomba de presión, aplicándose la técnica de presurizaciones repetidas (Hinckley et al., 1980).

Se realizaron entre 10 y 14 presurizaciones en cada planta, obteniéndose al menos 5 puntos en la porción linear de las curvas de $1 / \Psi_{\mathrm{a}}$ vs. CRA.

Las regresiones de las curvas de presión-volumen se analizaron a través de un programa de análisis elaborado por Schulte (versión 5.2, 8-12-88; cf. Schulte y Hinckley, 1985), el cual ajusta las curvas en las porciones lineales y no lineales de los datos y permite el cálculo del potencial osmótico a turgencia plena $\left(\Psi_{\pi_{100}}\right)$, el contenido relativo de agua y el potencial osmótico en plasmólisis incipiente $\left(C R A_{0}\right.$ y $\Psi_{\pi_{0}}$, respectivamente), el módulo máximo de elasticidad $\left(\xi_{\max }\right)$, el agua apoplásmica $(\theta)$ y el potencial máximo de presión $\left(\Psi_{\text {p100 }}\right)$.

El ajuste osmótico (AO) se calculó como la diferencia de $\Psi_{\pi_{100}}$ de plantas estresadas y plantas control, mientras que el ajuste elástico ( $\mathrm{AE}$ ) de manera análoga considerando los valores promedio de $\xi_{\max }$.

La evaluación del comportamiento de las plantas en respuesta a las bajas temperaturas se realizó indirectamente aplicando una metodología similar a la planteada por Close et al. (2002) que establece una evaluación cualitativa del nivel de pigmentación de las hojas por presencia de antocianas en los tejidos foliares (bajo: color verde, medio: color verde rojizo, alto: rojo), valores correlacionados con la severidad de la fotoinhibición inducida por frío.

Se midió periódicamente la altura y el diámetro basal de las plantas con cinta métrica y calibre digital, respectivamente. Se calculó la tasa de crecimiento relativo (RGR) en diámetro y altura, utilizando la fórmula de (Hunt, 1990):

$$
R G R=\left(\ln V_{2}-\ln V_{1}\right) / t_{2}-t_{1}
$$

Donde: $\quad V_{1}$ y $V_{2}$ son los valores de variable analizada al comienzo y al final del período de evaluación considerado

$t_{1} y t_{2}$ son las fechas de medición.

Para comparar las respuestas de las especies, es decir determinar el grado de cambio de las características medidas en diferentes unidades, se calculó un índice de Plasticidad para algunas variables fisiológicas y morfológicas.

Este índice, que varía entre 0 y 1 , se calcula a partir de la diferencia entre el valor máximo medio y mínimo medio que asume cada variable sobre el valor máximo (Valladares et al., 2002).

Los datos se analizaron utilizando un ANOVA multifactorial; considerando los efectos de especie (2), nivel de fertilización potásica (2) y régimen hídrico (3).

Se utilizó la prueba de Bartlett para testear la homogeneidad de varianza y se realizaron transformaciones en los casos en que fue necesario.

Las medias se separaron a través de la prueba de Tukey. Los análisis de procesaron con SAS statistical package (SAS Institute, Cary, NC). 


\section{RESULTADOS}

La sequía modificó la mayoría de las características evaluadas en este trabajo, disminuyendo las tasas de crecimiento y el tamaño final de las plantas de eucalipto. Se detectaron interacciones entre los factores considerados, por lo cual en ciertos casos se observaron diferencias en el comportamiento de las especies estudiadas.

\section{Características Fisiológicas}

En la medición realizada a fines de noviembre de 2009 se observó que las plantas que crecían bajo los niveles de restricción hídrica $\mathrm{S} 1$ y S2 mostraron menores valores promedio de CRA que las $C(p<0,0001)$. (Figura $\mathrm{N}^{\circ} 1 \mathrm{~A}$ ).

Paralelamente, el CRA de las $\mathrm{K} 1$ superó significativamente al de las $\mathrm{K} 0 \quad(p=0,0035)$

En concordancia con las respuestas previas, la $\mathrm{g}_{\mathrm{s}}$ se modificó en respuesta a ambos factores, fue superior en las plantas $C(p<0,0001)$ que en las sometidas a ciclos de sequía y con una tendencia marginalmente significativa $(p=0,08)$.

Se observaron diferencias entre las plantas de los niveles $\mathrm{K} 0$ y $\mathrm{K} 1$ en $E$. globulus, mostrando las últimas valores superiores de $\mathrm{g}_{\mathrm{s}}$ (Figura $\mathrm{N}^{\circ} 1 \mathrm{~B}$ ).
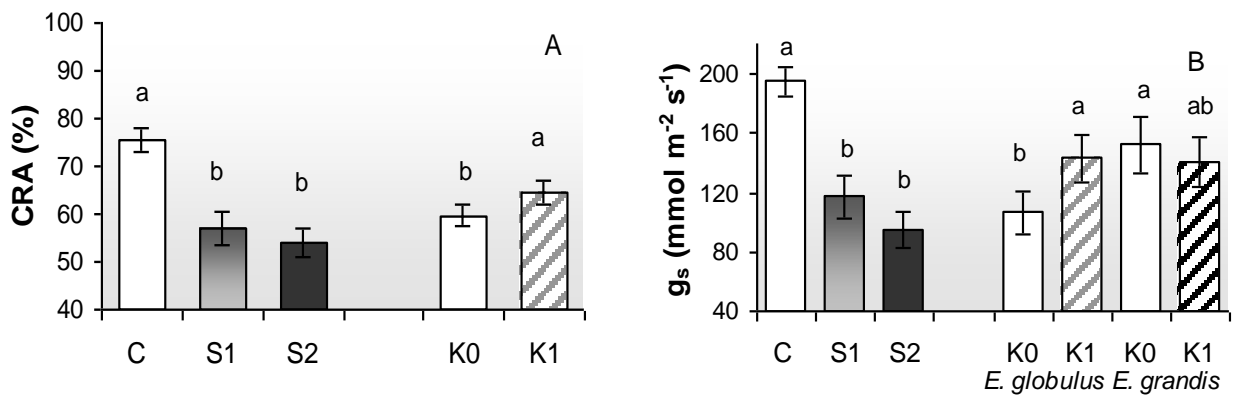

Valores promedio \pm un error estándar.

Letras distintas indican diferencias significativas $(p<0.05)$.

$\mathrm{C}$ : riego a diario, S1: riego cada 6 días y S2: riego cada 9 días. K0: sin fertilización y K1: con fertilización potásica.

Figura $N^{\circ} 1$

CONTENIDO RELATIVO DE AGUA (CRA) SEGÚN RÉGIMEN HÍDRICO Y NIVEL DE FERTILIZACIÓN POTÁSICA (A) Y CONDUCTANCIA ESTOMÁTICA $\left(\mathrm{g}_{\mathrm{s}}\right)$ SEGÚN RÉGIMEN HÍDRICO Y LA INTERACCIÓN ESPECIE X FERTILIZACIÓN POTÁSICA A FINES DE NOVIEMBRE DE 2009.

Al finalizar el experimento, el estado hídrico de las plantas al amanecer, a través de mediciones de $\Psi_{a}$ y el CRA se presenta en el Cuadro $N^{\circ} 2$. Las plantas S1 y S2 presentaron menores valores promedio de $\Psi_{\text {a }}$ que las $\mathrm{C}$, pero en contraste, solo las plantas S2 presentaron menores valores de CRA, diferenciándose de las $\mathrm{S} 1$ y las $\mathrm{C}$.

Al mediodía y para ambas variables, las interacciones entre régimen hídrico y la fertilización potásica fueron significativas. El potasio generó un efecto significativo en el estado 
hídrico de las plantas. Para el $\Psi_{\mathrm{a}}(p=0,0058)$ el efecto del $\mathrm{K}$ se produjo a nivel de las plantas $\mathrm{S} 2$. Con relación al CRA ( $p=0,0087)$, los valores en las plantas $S 1 K 1$ y S2K1 fueron más elevados que los de las S1K0 y la S2K0 y no se diferenciaron de las plantas C (Figura $N^{\circ} 2$ ). La gs solo disminuyó en las plantas $S 2(p=0.0327)$, pero en esta oportunidad no se observó efecto de la fertilización con $\mathrm{K}$.

\section{Cuadro $\mathrm{N}^{\circ} 2$ \\ VALORES DE POTENCIAL AGUA $\left(\Psi_{\mathrm{a}}\right)$ Y CONTENIDO RELATIVO DE AGUA (CRA) AL AMANECER SEGÚN RÉGIMEN HÍDRICO AL FINAL DEL EXPERIMENTO}

\begin{tabular}{|c|c|c|}
\hline $\begin{array}{c}\text { Régimen } \\
\text { Hídrico }\end{array}$ & $\begin{array}{c}\boldsymbol{\Psi}_{\mathrm{a}} \\
\mathbf{( M P a}) \\
\boldsymbol{p}\end{array}$ & $\begin{array}{c}\text { CRA } \\
(\%)\end{array}$ \\
\hline & $\mathbf{0 , 0 0 5 6}$ & $\mathbf{0 , 0 0 7 1}$ \\
$\mathrm{C}$ & $-0,22 \pm$ & $81,02 \pm 1,33$ \\
$\mathrm{~S} 1$ & $0,014 \mathrm{a}$ & $\mathrm{a}$ \\
$\mathrm{S} 2$ & $-0,40 \pm$ & $82,67 \pm 1,59$ \\
& $0,021 \mathrm{~b}$ & $\mathrm{a}$ \\
& $-0,45 \pm$ & $77,21 \pm 1,10$ \\
\hline
\end{tabular}

Valores promedio \pm un error estándar.

Letras distintas indican diferencias significativas $(p<0,05)$.

C: riego diario, S1: riego cada 6 días y S2: riego cada 9 días.
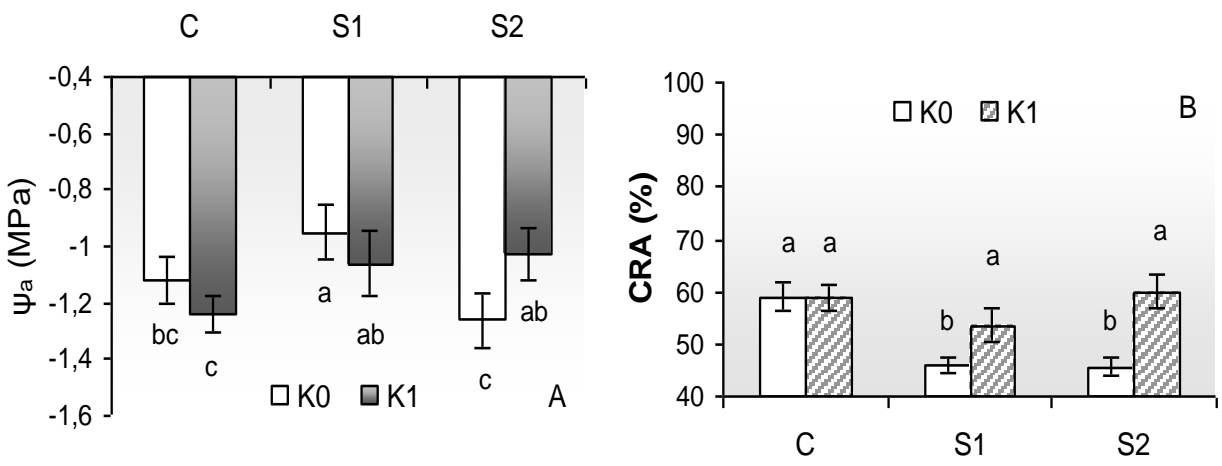

Valores promedio \pm un error estándar.

Letras distintas indican diferencias significativas $(p<0.05)$.

C: riego a diario, S1: riego cada 6 días y S2: riego cada 9 días. K0: sin fertilización y K1: con fertilización potásica.

\section{Figura $\mathrm{N}^{\circ} 2$}

POTENCIAL AGUA ( $\Psi_{\mathrm{a}}$ ) (A) Y CONTENIDO RELATIVO DE AGUA (CRA) (B) AL MEDIODÍA SEGÚN LA INTERACCIÓN RÉGIMEN HÍDRICO X FERTILIZACIÓN POTÁSICA AL FINAL DEL EXPERIMENTO.

En respuesta a los ciclos de sequía se produjo un descenso en el $\Psi_{\pi_{100}}$ y un aumento en el $\xi_{\max }$ de las plantas de eucalipto. La magnitud promedio de AO en las plantas S1 y S2 fue 0,106 $\mathrm{MPa}$ y 0,201 $\mathrm{MPa}$, respectivamente (Figura $\mathrm{N}^{\circ} 3 \mathrm{~A}$ ). En contraste, solo las plantas $\mathrm{S} 2$ mostraron un incremento significativo en el $\xi_{\max }$ de 2,2 MPa (Figura $\mathrm{N}^{\circ} 3 \mathrm{~B}$ ). Paralelamente, E. grandis mostró menor $\Psi_{\pi_{100}}$ y mayor $\xi_{\max }$ que E. globulus (Figuras $\mathrm{N}^{\circ} 3 \mathrm{~A}$ y $3 \mathrm{~B}$ ). 

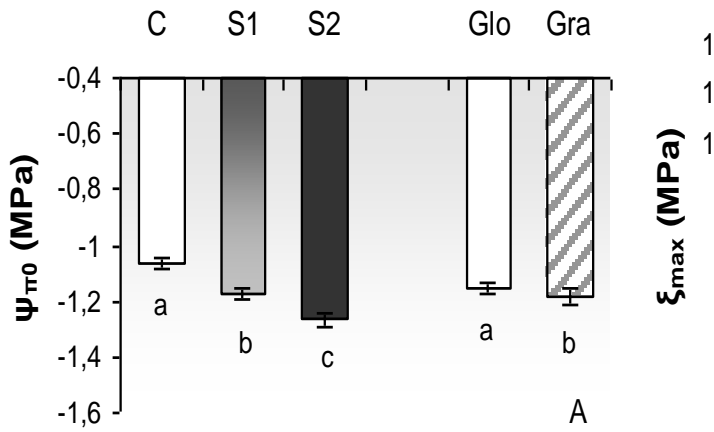

Valores promedio \pm un error estándar.

Letras distintas indican diferencias significativas $(p<0.05)$.

$\mathrm{C}$ : riego a diario, S1: riego cada 6 días y S2: riego cada 9 días.

Glo: E. globulus y Gra: E. grandis.

Figura $\mathrm{N}^{\circ} 3$

POTENCIAL OSMÓTICO A SATURACIÓN $\left(\Psi_{\pi_{100}}\right)(A)$ Y MÓDULO MÁXIMO DE ELASTICIDAD $\left(\xi_{\max }\right)(B)$ SEGÚN RÉGIMEN HÍDRICO Y ESPECIE AL FINAL DEL EXPERIMENTO

Análisis realizados para cada una de las especies en forma separada, mostraron interacciones significativas entre régimen hídrico y fertilización potásica (Cuadro $\mathrm{N}^{\circ} 3$ ). En $E$. globulus, las plantas de los niveles S1 y S2 mostraron un descenso significativo en el $\Psi_{\pi_{100}}$ y particularmente en las del nivel S2, el agregado de $\mathrm{K}$ generó un descenso adicional significativo en el $\Psi_{\pi_{100}}$ en las plantas $S 2(p=0,05)$. En E. grandis se observó descenso significativo en las plantas correspondientes a los tratamientos S1K1 como así mismo en las S2K0 y S2K1. Es decir, el agregado de $\mathrm{K}$ también produjo un descenso en el $\Psi_{\pi_{100}}(p=0,048)$.

\section{Cuadro $\mathrm{N}^{\circ} 3$}

VALORES DE POTENCIAL OSMÓTICO A SATURACIÓN $\left(\Psi_{\pi_{100}}\right)$

\section{SEGÚN LA INTERACCIÓN RÉGIMEN HÍDRICO X FERTILIZACIÓN POTÁSICA.}

\begin{tabular}{|c|c|c|}
\hline \multirow{2}{*}{ Tratamiento } & \multicolumn{2}{|c|}{$\Psi_{\pi_{100}}(\mathrm{MPa})$} \\
\cline { 2 - 3 } & E. globulus & E. grandis \\
\hline CK0 & $-1,00 \pm 0,051 \mathrm{a}$ & $-1,15 \pm 0,022 \mathrm{ab}$ \\
K1 & $-1,05 \pm 0,030 \mathrm{ab}$ & $-1,06 \pm 0,053 \mathrm{a}$ \\
S1 K0 & $-1,17 \pm 0,024 \mathrm{C}$ & $-1,17 \pm 0,035 \mathrm{~b}$ \\
K1 & $-1,08 \pm 0,026 \mathrm{bc}$ & $-1,26 \pm 0,038 \mathrm{~cd}$ \\
S2K0 & $-1,23 \pm 0,022 \mathrm{c}$ & $-1,22 \pm 0,025 \mathrm{bc}$ \\
K1 & $-1,30 \pm 0,055 \mathrm{~d}$ & $-1,33 \pm 0,069 \mathrm{~d}$ \\
\hline
\end{tabular}

Valores promedio \pm un error estándar.

Letras distintas indican diferencias significativas $(p<0,05)$.

C: riego diario, S1: riego cada 6 días y S2: riego cada 9 días.

En E. grandis los niveles más altos de antocianas se presentaron en las plantas C (follaje de color rojizo), mientras que las plantas $\mathrm{S} 1$ y S2 presentaron nivel medio y bajo, respectivamente. El follaje de E. globulus presentó bajos niveles de coloración rojiza y no se observaron diferencias entre las plantas de los tres regímenes hídricos. Tampoco se observaron diferencias entre las 
plantas $\mathrm{K} 0$ y K1. Las plantas no presentaron tejidos dañados y las condiciones experimentales no generaron muerte de individuos.

\section{Variables Morfológicas}

Las tasas de crecimiento relativo en diámetro y altura se redujeron por efecto del régimen hídrico. Al mismo tiempo las especies mostraron valores promedio diferentes en diámetro (Cuadro $\left.\mathrm{N}^{\circ} 4\right)$.

\section{Cuadro $\mathrm{N}^{\circ} 4$ \\ TASAS DE CRECIMIENTO RELATIVO (TCR) EN DIÁMETRO Y ALTURA SEGÚN RÉGIMEN HÍDRICO Y ESPECIE}

\begin{tabular}{|c|c|c|}
\hline $\begin{array}{c}\text { Régimen Hídrico } \\
p\end{array}$ & $\begin{array}{c}\text { TCR en Diámetro } \\
\left(\mathrm{mm} \mathrm{mm}^{-1} \mathrm{~d}^{-1}\right) \\
<0,0001\end{array}$ & $\begin{array}{c}\text { TCR en Altura } \\
\left(\mathrm{cm} \mathrm{cm}^{-1} \mathrm{~d}^{-1}\right) \\
0,0001\end{array}$ \\
\hline $\begin{array}{l}\mathrm{C} \\
\mathrm{S} 1 \\
\mathrm{~S} 2\end{array}$ & $\begin{array}{l}0,0057 \pm 0,00029 \mathrm{a} \\
0,0051 \pm 0,00020 \mathrm{a} \\
0,0040 \pm 0,00018 \mathrm{~b}\end{array}$ & $\begin{array}{l}0,0044 \pm 0,00018 a \\
0,0038 \pm 0,00022 b \\
0,0033 \pm 0,00019 b\end{array}$ \\
\hline $\begin{array}{c}\text { Especie } \\
p\end{array}$ & 0,0014 & 0,099 \\
\hline $\begin{array}{l}\text { E. globulus } \\
\text { E. grandis }\end{array}$ & $\begin{array}{l}0,0045 \pm 0,00019 b \\
0,0054 \pm 0,00023 a\end{array}$ & $\begin{array}{l}0,0036 \pm 0,00017 \\
0,0039 \pm 0,00019\end{array}$ \\
\hline
\end{tabular}

Valores promedio \pm un error estándar.

Letras distintas indican diferencias significativas $(p>0,05)$.

$\mathrm{C}$ : riego a diario, S1: riego cada 6 días y S2: riego cada 9 días.

El diámetro final se redujo en forma creciente en las plantas $S 1$ y $S 2(p<0,001)$, pero la altura se modificó significativamente en forma similar en ambos grupos de plantas $(p<0,001)$. Al mismo tiempo se observó en $E$. grandis menor altura $(p<0,001)$ que en $E$. globulus, pero diámetro similar $(p>0,05)$ (Figura $\left.N^{\circ} 4\right)$.
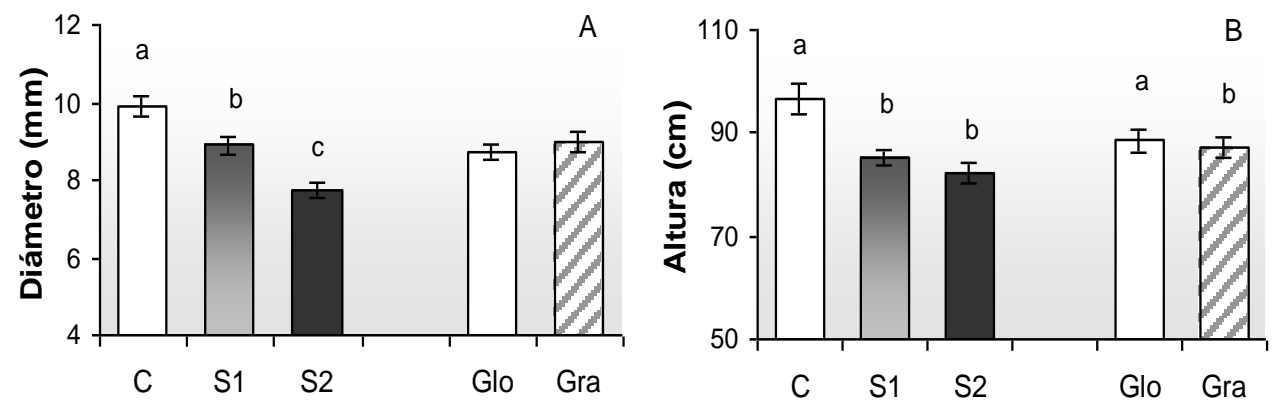

Valores promedio \pm un error estándar.

Letras distintas indican diferencias significativas $(p<0.05)$.

$\mathrm{C}$ : riego a diario, S1: riego cada 6 días y S2: riego cada 9 días.

Glo: E. globulus y Gra: E. grandis.

Figura $\mathrm{N}^{\circ} 4$

DIÁMETRO Y ALTURA SEGÚN RÉGIMEN HÍDRICO Y ESPECIE AL FINAL DEL EXPERIMENTO 


\section{Índices de Plasticidad}

Los índices de plasticidad para las características seleccionadas mostraron que el crecimiento en altura fue más afectado por la sequía que el crecimiento en diámetro y también se observaron mayores reducciones en las variables fisiológicas y menores en el crecimiento de $E$. globulus con relación a $E$. grandis.

\section{Cuadro $\mathrm{N}^{\circ} 5$ \\ INDICES DE PLASTICIDAD PARA LAS DIVERSAS CARACTERÍSTICAS CONSIDERADAS}

\begin{tabular}{|c|cccccccccc|}
\hline \multirow{2}{*}{ Tratamiento } & \multicolumn{9}{|c}{ E globulus } & \multicolumn{9}{c|}{ E grandis } \\
& $\mathbf{g}_{\mathbf{s}}$ & $\boldsymbol{\Psi}_{\pi_{100}}$ & $\boldsymbol{\xi}_{\max }$ & Diámetro & Altura & $\mathbf{g}_{\mathbf{s}}$ & $\boldsymbol{\Psi}_{\pi_{100}}$ & $\boldsymbol{\xi}_{\max }$ & Diámetro & Altura \\
\hline CK1 & 0,121 & 0,051 & 0,033 & 0,082 & 0,005 & 0,058 & 0,078 & 0,240 & 0,021 & 0,198 \\
S1K0 & 0,620 & 0,144 & 0,085 & 0,082 & 0,152 & 0,128 & 0,017 & 0,062 & 0,111 & 0,158 \\
K1 & 0,403 & 0,077 & 0,022 & 0,038 & 0,146 & 0,343 & 0,089 & 0,004 & 0,114 & 0,186 \\
S2K0 & 0,580 & 0,183 & 0,165 & 0,216 & 0,222 & 0,522 & 0,058 & 0,097 & 0,253 & 0,226 \\
K1 & 0,315 & 0,229 & 0,196 & 0,142 & 0,146 & 0,550 & 0,013 & 0,068 & 0,206 & 0,166 \\
\hline Promedio & 0,408 & 0,137 & 0,100 & 0,112 & 0,134 & 0,032 & 0,075 & 0,094 & 0,141 & 0,187 \\
\hline
\end{tabular}

\section{DISCUSIÓN Y CONCLUSIONES}

Estos resultados indican que las dos especies manifestaron cambios significativos en la mayoría de los caracteres estudiados cuando estuvieron sometidas a condiciones de estrés, es decir experimentaron un proceso de aclimatación. Se observó una reducción en la $\mathrm{g}_{\mathrm{s}}$ de las plantas, estrategia para evitar las condiciones de sequía, que tiene un importante impacto sobre la supervivencia y el crecimiento de las plantas en ambientes con deficiencias hídricas ya que previene que las hojas y tallos alcancen valores de contenido hídrico que dañen irreversiblemente a los tejidos. Aún cuando la reducción en la gs representa una limitación para la asimilación de $\mathrm{CO}_{2}$ y en consecuencia para la acumulación de biomasa, las plantas evidenciaron paralelamente capacidad para desarrollar $\mathrm{AO}$, mecanismo de tolerancia a la sequía. El AO generado por el descenso en el $\Psi_{\pi_{100}}$ como consecuencia de la acumulación de solutos disminuye el $\Psi_{\text {a }}$ permitiendo mantener el gradiente de $\Psi$ entre la planta y el suelo a medida que disminuye el contenido hídrico de este, por lo tanto se mantiene la absorción de agua del suelo, se facilita la turgencia de los tejidos y por consiguiente los procesos fisiológicos dependientes de dicha condición. El aumento en el $\xi_{\max }$, que implica una disminución en la elasticidad de la pared celular, favorece, al igual que el $\mathrm{AO}$, el mantenimiento del gradiente de $\Psi_{\mathrm{a}}$ entre la planta y el suelo, y por lo tanto la absorción de agua a bajos contenidos hídricos del suelo.

Estas respuestas frente a la sequía han sido reportadas en estudios previos en eucalipto (Tuomela 1997, Guarnaschelli 2009, Merchant et al., 2007). Cabe destacar que para algunas variables la magnitud de los cambios se correlacionó con el nivel de severidad del estrés hídrico al cual estuvieron sometidas las plantas (Guarnaschelli et al., 2003).

El agregado de $\mathrm{K}$ tuvo un efecto positivo en las plantas dado que contribuyó a un descenso adicional en el $\Psi_{\pi 100}$, protegiendo a las plantas que crecían bajo sequía. Estos resultados confirman nuevamente estudios previos donde se observó que plantas de E. globulus fertilizadas con $\mathrm{K}^{+}$presentaban menor $\Psi_{\pi}$ que las plantas sin aporte adicional de $\mathrm{K}^{+}$, lo cual generó un aumento en la tolerancia al déficit hídrico (Guarnaschelli et al., 2010). En este trabajo un similar efecto se observó también en las plantas de E. grandis.

No se observaron daños en las plantas derivados de la exposición a las bajas 
temperaturas, pero en contraste se presentaron diferencias en la coloración del follaje entre especies y entre regímenes hídricos. El follaje de las plantas C de $E$. grandis presentó una tonalidad mas rojiza con relación al presente en las plantas sometidas a ciclos de sequía, que resultó mas verdoso. Las tonalidades más rojizas son derivadas de la presencia de antocianas, compuesto asociados con la fotoprotección de las plantas en condiciones de bajas temperaturas. Estas respuestas indican que las plantas bajo sequía mostraron menores niveles de fotoinhibición y sugieren que los ajustes en la tolerancia a la sequía las habrían protegido de las bajas temperaturas.

Costa e Silva et al. (2009) plantearon que las plantas de E. globulus más tolerantes a la sequía resultaron más resistentes a las bajas temperaturas y asociaron esas respuestas con la mayor capacidad de ajuste osmótico. De hecho, la acumulación de solutos contribuye no solamente a la aclimatación de las plantas frente a la sequía, estos actúan también como crioprotectores, facilitando la aclimatación a las bajas temperaturas (Kozlowski y Pallardy 2002). Coopman et al. (2010) observaron que la resistencia al frío depende del genotipo y de los tratamientos de aclimatación a la sequía y destacaron la importancia de aplicarlos en vivero previo a la plantación. Los resultados obtenidos nuevamente avalan la efectividad de los tratamientos de aclimatación a la sequía sobre la tolerancia a la sequía y a las bajas temperaturas en E. globulus y aportan información novedosa sobre su efectividad en plantas de E. grandis.

El $\mathrm{K}$ es el principal catión osmorregulador y su aporte a las plantas de E. globulus y $E$. grandis, como se adelantara previamente, contribuyó al descenso en el $\Psi_{\pi_{100}}$ y al aumento en la tolerancia a la sequía. No obstante, no fue posible establecer su efecto sobre la protección frente a las bajas temperaturas. Teniendo que en cuenta que en este trabajo solo se consideró a la presencia de antocianas como variable indicadora de las respuestas frente a las bajas temperaturas, el hecho de no haber encontrado mayores diferencias entre niveles de fertilización, no implica que el $\mathrm{K}$ no haya ejercido un efecto positivo, sino que no fue posible detectarlo bajo las condiciones experimentales impuestas. Se plantea la necesidad de realizar nuevos experimentos realizando pruebas de "fuga de electrolitos" (Electrolyte leakage), técnica de gran eficiencia y rapidez (Ritchie y Landis 2010), cuyos resultados muestran buenos correlaciones con la performance de las plantas a campo (Radoglou y Raftoyannis, 2001; Bigras y Dumais, 2005).

Otro aspecto importante derivado del tratamiento de fertilización es que las plantas estresadas del nivel K1 mostraron mayor contenido de agua, lo que implica que la fertilización con $\mathrm{K}$ es una herramienta útil para los productores de Eucalyptus, relacionada con la calidad de las mismas y con potencial efecto positivo luego de la plantación.

Finalmente, los ajustes plásticos experimentados en los caracteres relacionados con la resistencia al estrés permitieron la aclimatación de las plantas. Los índices de plasticidad mostraron un ligero mayor ajuste en los caracteres fisiológicos de $E$. globulus y si bien no se produjo mortalidad de ejemplares, las tasas de crecimiento relativo disminuyeron por efecto de la sequía y las bajas temperaturas en ambas especies, pero en $E$. globulus la reducción fue menor que la observada en $E$ grandis.

\section{REFERENCIAS}

Beadle, C. L.; Ludlow, M. M. P. y Honeysett, J. L., 1993. Water relations. Páginas 113-128. En D. O. Hall, J. M. O. Scurlock, H. R. Bolhar-Nordencampf, R. C. Leegood y S. P. Long, editores. Photosynthesis and production in a changing environment: A field and laboratory manual, Chapman and Hall, London.

Bigras, F. J. y Dumais, D., 2005. Root-freezing damage in the containerized nursery: Impact on plantation sitesA review. New Forests 30: 167-184.

Burguess, I. P., 1983. Tolerance of two years old Eucalyptus grandis and Eucalyptus saligna to winter frost in Canberra. IUFRO-AFOCEL- Coloque international sur les Eucalyptus résistants au froid. Bordeaux, Francia. 2630/9/1983. Páginas 356-386. 
Cakman, I., 2005. The role of potassium in alleviating detrimental effects of abiotic stresses in plants. Journal of Plant Nutrition and Soil Science 168: 521-530.

Close, D. C.; Beadle, C. L.; Holz, G. K. y Brown, P. H., 2002. Effects of shadecloth tree shelter on cold-induced photoinhinition, foliar anthocyanin and growth of Eucalyptus globulus and $E$. nitens seedlings during establishment. Australian Journal of Botany 50: 15-20.

Coopman, R. E.; Jara, J. C.; Escobar, R., Corcuera, L. J. y Bravo, L. A., 2010. Genotypic variation in morphology and freezing resistance of Eucalyptus globulus seedlings subjected to drought hardening in nursery. Electronic Journal of Biotechnology 13, 10.

Costa e Silva, F.; Shvaleva, A.; Maroco, J. P.; Almeida, M. H.; Chaves, M. M. y Pereira, J. S., 2004. Responses to water stress in two Eucalyptus globulus clones differing in drought tolerance. Tree Physiology 24 : 1165-1172.

Costa e Silva, F. A.; Shvaleva, A.; Broetto, F.; Ortuño, M. F.; Rodrigues, M. L.; Almeida, M. H.; Chaves, M. M. y J. S. Pereira, J. S., 2009. Acclimation to short-term low temperatures in two Eucalyptus globulus clones with contrasting drought resistance. Tree Physiology 29:77-86.

Garrán, S. M.; Garín, R. O. y Marcó, M. A., 2007. El clima de la región de Concordia y sus heladas. En Actas XXII Jornadas Forestales de Entre Ríos. Concordia, Entre Ríos, 25-26/10/2007.

Garrán, S. M. y Garín, R. O., 2012. Informe heladas del 7, 8 y 9 de Junio en la región citrícola del Río Uruguay

Garau, A. M.; Guarnaschelli, A. B.; Mema, V. y Lemcoff, J. H., 2004a. Tissue water relations in Eucalyptus seedlings: Effects of species, $\mathrm{K}$ fertilization and drought. En CD Conferencia Internacional IUFRO Eucalyptus in a Changing World. Aveiro, Portugal. 11-15/10/2004.

Garau, A. M.; Guarnaschelli, A. B.; Prystupa, P. y Lemcoff, J. H., 2004b. Relaciones hídricas en plantines de Eucalyptus sp. Sujetos a la interacción de fertilización nitrogenada y potásica con restricción hídrica. En Actas XXV Reunión Argentina de Fisiología Vegetal. San Rosa, La Pampa. 22-24/9/2004.

Golfari, L., 1985. Distribución regional y condiciones ecológicas de los eucaliptos cultivados en la Argentina. Problemas inherentes. C.I.E.F. Publicación técnica № 1.19 páginas.

Gould, K. S., 2004. Nature's Swiss army knife: The diverse protective roles of anthocyanins in leaves. Journal of Biomedicine \& Biotechnology 5: 314-320.

Guarnaschelli, A. B., 2009. Efecto del ajuste osmótico y la elasticidad de la pared celular sobre el crecimiento de Eucalyptus globulus durante el establecimiento. Tesis M.Sc., Facultad de Agronomía, Universidad de Buenos Aires, Argentina.

Guarnaschelli, A. B.; Lemcoff, J. H.; Prystupa, P. y Basci, S. O., 2003. Responses to drought preconditioning in Eucalyptus globulus Labill. provenances. Trees 17: 501-509.

Guarnaschelli, A. B.; Prystupa, P. y Lemcoff, J. H., 2006. Drought conditioning improves water status, stomatal conductance and survival of Eucalyptus globulus ssp. bicostata. Annals of Forest Science 63: 941-950.

Guarnaschelli, A. B.; Ruiz Nuñez, J.; Chiavassa, J. A.; Fedotova, N. y Garau, A. M., 2010. Aclimatación en vivero en plantas de Eucalyptus por restricción hídrica y fertilización potásica. En CD XXIV Jornadas Forestales de Entre Ríos. Concordia, Entre Ríos. 28-29/10/2010.

Harrand, L., 2005. La Utilización de Híbridos Interespecíficos como Alternativa Forestal. Revista IDIA XXI: № 8 , págs. 165-168.

Harrand, L. y Marcó, M. A., 2012. Investigación hacia el desarrollo de material genético tolerante al frío. En Actas XXVI Jornadas Forestales de Entre Ríos. Concordia, Entre Ríos, 4-5/10/2012. 8 páginas.

Hinckley, T. M.; Duhme, F.; Hinckley, A. R. y Richer, H., 1980. Water relations of drought hardy shrubs: Omotic potential and stomatal reactivity. Plant, Cell and Environment 3: 131-140.

Hughes, N. M.; Neufeld, H. S. y Burkey, K. O., 2005. Functional role of anthocyanins in high-light winter leaves of the evergreen herb Galax urceolata. New Phytologist 168: 575-587 
Hunt, R., 1990. Basic growth analysis. Plant growth analysis for beginners. Unwin Hyman Ltd., London. 112 páginas.

INTA, 2012. Las heladas del 7, 8 y 9 de Junio en la costa del Río Uruguay y sus efectos sobre las plantaciones de eucaliptos. 11 páginas.

IPCC, 2007. Fourth Assessment Report: Climate Change Working Group I Report. The Physical Science Basis. Cambridge University Press, Cambridge, New York, NY, USA.

Kozlowski, T. T. y Pallardy, S. G., 2002. Acclimation and adaptive responses of woody plants to environmental stresses. Botanical Review 68: 270-334.

Landis. T. D., 2005. Macronutrients. Potassium. Forest Nursery Notes. Winter 2005. Págs. 5-11.

Landis, T. D. ; Tinus, R. W. ; Mc Donald, S. E. y Barnett, J. P., 1989. The Container Tree Nursery Manual, Vol. 4, Seedling nutrition and irrigation, U.S.D.A. Forest Service. Agricultural Handbook 674. Washington D.C.

Larocca, F., 2012. Daños por heladas en plantaciones de Eucalyptus grandis, posibles herramientas desde el manejo. En Actas XXVI Jornadas Forestales de Entre Ríos. Concordia, Entre Ríos, 4-5/10/2012. 10 páginas.

Marcó, M. A., 1986. Tolerancia a heladas en orígenes y procedencias de Eucalyptus grandis. Información Forestal. EEA INTA Concordia. 5 págs.

Marcó, M. A., 2005. Conceptos generales de mejoramiento genético forestal y su aplicación a los bosques cultivados de la Argentina. Págs. 9-17, en C. A. Norberto, editor. Mejores árboles para más forestadores: El programa de producción de material de propagación mejorado y el mejoramiento genético en el proyecto forestal de desarrollo. SAGPyA. Buenos Aires.

Merchant, A.; Callister, A.; Ardnt, S.; Tausz, M. y Adams, M., 2007. Contrasting physiological responses of six Eucalyptus species to water deficits. Annals of Botany 100:1507-1515.

Moraga, P.; Escobar, R. y Valenzuela, S., 2006. Resistance to freezing in three Eucalyptus globulus Labill. subspecies. Electronic Journal of Biotechnology 9: 310-314. http://www.ejbiotechnology.info/index.php/ejbiotechnology/index.

Morgan, J. M., 1984. Osmoregulation and water stress in higher plants. Annual Review of Plant Physiology 35:299-319.

Nicotra, A. B.; Hofman, M.; Siebke, K. y Ball, M. C., 2003. Spatial patterning of pigmentation in evergreen leaves in response to freezing stress. Plant, Cell and Environment 26: 1983-1904.

Pita, P. y Pardos, J. A., 2001. Growth, morphology, water use and tissue water relations of Eucalyptus globulus clones in response to water deficit. Tree Physiology 21:599-507.

Radoglou, K. y Raftoyannis, Y., 2001. Effects of desiccation and freezing on vitality and field performance of Broad leaves tree Species. Annals of Forest Science 58: 59-68.

Raymond, C. A.; Owen, J. V.; Eldridge, G. G. y Harwood, C. E., 1992. Screening Eucalypts for frost tolerance in brreding programs. Canadian Journal of Forest Research 22: 1271-1277.

Richter, H., 1978. A diagram for the description of water relations of plants cells and organs. Journal of Experimental Botany 29: 1197-1203.

Ritchie, G. A y Landis, T. D., 2010. Assessing Plant Quality. En Landis et al., Editores. The Container Tree Nursery Manual. Volumen 7. Capítulo 2. 80 págs.

Römheld, V. y Kirkby, E. A., 2010. Research on potassium in agriculture: Needs and Prospects. Plant and Soil 335: $155-180$.

S.A.S Institute, 2000. SAS/STAT Guide for personal computer. Cary. N.C.

Schulte, P. y Hinckley, T., 1985. A comparison of pressure-volume curve data analysis techniques. Journal of Experimental Botany 36: 1590-1602. 
Smith, D. M.; Larson, B. C.; Kelty, M. J. y Ashton, P. M. S., 1997. The practice of Silviculture: Applied Forest Ecology. John Wiley \& Sons Inc. New York, 537 Págs.

Stoneman, G. L., 1994. Ecology and physiology of establishment of Eucalyptus seedlings from seed: A review. Australian Forestry 57: 11-30.

Texeira, P. C.; Moraes Gonçalvez, J. L.; Artur Junior. J. C. y Dezordi, C., 2008. Eucalyptus sp. seedling response to potassium fertilization and soil water. Ciencia Florestal 18: 47-63.

Tuomela, K., 1997. Leaf water relations in six provenances of Eucalyptus microtheca: A greenhouse experiment. Forest Ecology and Management 92: 1-10.

Van den Driessche, R., 1992. Changes in drought resistance and root growth capacity of container seedlings in response to nursery drought, nitrogen and potassium treatments. Canadian Journal of Forest Research 22: 740-749.

Valladares, F.; Chico, J. M.; Aranda, I.; Balaguer, L.; Dizengremel, P.; Manrique, E. y Dreyer, E., 2002. The greater seedling high-light tolerance of Quercus robur over Fagus sylvatica is linked to a greater physiological plasticity. Trees 16: 395-403.

Volker, P. W.; Owen, J. B. y Borralho, N. M. G., 1995. Genetic variances and covariances for frost tolerance in Eucalyptus globulus and Eucalyptus nitens. Silvae Genetica 43: 366-372

White, D. A.; O'Grady, A. P.; Battaglia, M.; Bruce, J. L. y Pinkard, E. A., 2011. Drought responses of Eucalyptus plantations - from physiology to management. En Meeting Abstract IUFRO Tree Biotechnology Conference 2011: From genomes to integration and delivery. Arraial d'Ajuda, Bahia, Brasil. 26/6-2/7/2011. 3 páginas. 
